\title{
Role of Substrate Flexibility on Cardiac Cell Culture Electrophysiological Properties
}

\author{
L Gómez-Cid ${ }^{1}$, L Fuentes ${ }^{1}$, I Hernández-Romero ${ }^{1}$, MS Guillem², F Atienza ${ }^{1}$, F Fernández-Avilés ${ }^{1}$, \\ AM Climent ${ }^{1}$ \\ ${ }^{1}$ Hospital Gregorio Marañón, Instituto de Investigación Sanitaría Gregorio Marañon, Madrid, Spain \\ ${ }^{2}$ ITACA, Universitat Politecnica de Valencia, Valencia, Spain
}

\begin{abstract}
Major considerations in the design of engineered cardiac patches are the regenerative and electrophysiological properties of cardiac cells. The objective of this study is to compare key cardiomyocyte functional properties for cardiac repair such as proliferation, migration and displacement, and conduction velocity of atrial murine cells (HL-1myocytes) in two different substrates: (1) rigid Petri dishes and (2) flexible PDMS (polydimethylsiloxane) wells. Results from this study confirm that the use of flexible substrates induces a more similar cardiac phenotype to that shown in vivo, especially characterized by higher conduction velocities. This result was supported by a higher expression of genes involved in action potential generation and propagation. Flexible membranes will allow further studying the effect of simultaneous mechanical and electrical stimulation on cardiac properties.
\end{abstract}

\section{Introduction}

Cardiovascular diseases are the first leading cause of death worldwide, being ischemic heart disease and myocardial infarction the most common [1]. If cardiomyocyte depletion occurs, it can cause further heart failure, as remodelling occurs with heterogeneous tissue. This tissue presents lower conduction velocities that provoke asynchronous electric conduction, thus increasing the risk of future re-entries that can lead to cardiac arrhythmias [2].

Engineered cardiac patches seem a promising tool to solve this problem. Furthermore, they allow adequate simulation of cardiac tissue dynamics in vitro to obtain models and realistic responses of pharmacological agents and therapies. To serve those purposes, cardiac patches must have optimal structural, mechanical and electrophysiological properties, and to populate them, cell proliferation, migration and displacement are also important. Myocardial tissue has complex architecture and cell interconnections, and to mimic the Extra Cellular Matrix (ECM) environment is one of the main challenges of tissue engineering, as it is crucial to obtain in vivo like phenotypes, such as higher conduction velocities and more efficient and synchronous contractions [3].

The purpose of this work is to study and quantify the effect of using flexible membranes as substrate on different properties that play a key role in cardiac patches development and functioning. The proposed flexible membranes used are wells made out of PDMS (polydimethylsiloxane). Its flexibility is closer to that of the ECM in contrast to conventional Petri dishes, providing a more in vivo-like environment. The use of these membranes will also offer the possibility of studying the impact of mechanical and electrical stimulation during cell culture, which may result in further enhancement of these desired properties.

\section{Methods}

\subsection{Cell culture}

Hl-1 cells were maintained, grown and proliferated according to the standard protocol established by Claycomb et al. [4].

\subsection{Proliferation assay}

To study proliferation of HL-1 cells, alamarBlue ${ }^{\circledR}$ (oxidation-reduction colorimetric indicator) was used. Cells were seeded in three p35s (Petri dishes) and three PDMS wells at an initial concentration of 35000 cells $/ \mathrm{cm}^{2}$ on day 1 and cultured with $208 \mu \mathrm{L}$ of medium $/ \mathrm{cm}^{2}$. From day 2 to day 5, equal amount of media was changed at 5 pm and incubated overnight. In the following morning, alamarBlue ${ }^{\circledR}$ was added at 9 am in a concentration of 1 $\mu \mathrm{L} / 10 \mu \mathrm{L}$ of medium and left in the incubator for 4 hours. After this time, $300 \mu \mathrm{L}$ of each sample (medium + alamarBlue $\AA$ ) were loaded into 96-well plates (100 $\mu \mathrm{L} /$ well, 3 replicates). Absorbance for each well was 
measured using EMax® Plus Microplate Reader from bioNova cientifica.

\subsection{Migration and displacement}

Migration and displacement properties of HL-1 cells were measured by quantifying the time needed by the cells to close an injury. Specifically, a linear wound was generated after reaching full confluence by scratching with the tip of a pipette in three p35s and three PDMS wells. After wound induction, cell migration and displacement was recorded using time-lapse equipment (Lumascope 400 iVue, etaluma) by taking an image every 5 minutes during 60 hours. To process those images, custom software in MATLAB (The MathWorks) was developed to obtain graphs of confluence over time in the wound area and displacement velocity values.

\subsection{Conduction velocity}

Four confluent samples of HL-1 on each substrate were employed to measure electrophysiological conduction velocity. Specifically, calcium transients were recorded using optical mapping technique, and results were further analysed by custom software developed in MATLAB to obtain impulse propagation velocity measurements [5].

Once electrical activity was confirmed, lines were made in the cell culture by scratching in order to allow electric conduction to happen only in a certain direction.

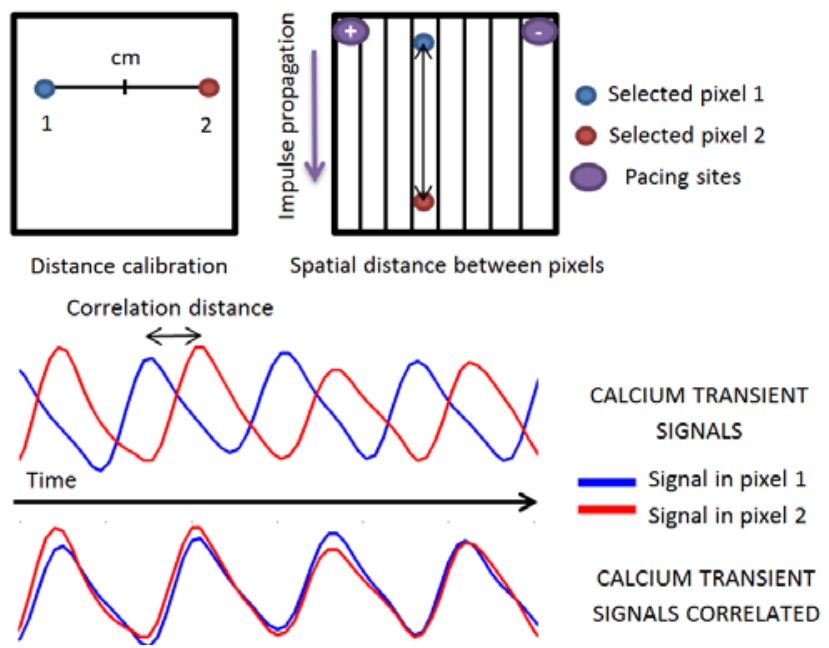

Figure 1. Process for calculating conduction velocity. (1) Calibration. (2) Pixel selection. (3) Correlation of fluorescence signal in time of pixel 1 and pixel 2 (4) Conduction velocity obtaining as Spatial distance (cm)/Correlation distance (s).

The movies were further processed in custom software developed in MATLAB. In order to obtain conduction velocity values, two pixels are selected and their two fluorescence signals along time are correlated. With a calibration distance (spatial information) previously indicated and the electric impulse correlation distance (temporal information), conduction velocity is calculated. This process can be observed in Figure 1.

Table 1. Primers used for reverse transcription polymerase chain reaction (RT-PCR).

\begin{tabular}{|c|c|c|c|}
\hline Gene & Protein & $\begin{array}{c}\text { Forward Primer (5'- } \\
>3 \text { ') }\end{array}$ & $\begin{array}{c}\text { Reverse Primer (5'- } \\
\text { >3’) }\end{array}$ \\
\hline SCN5A & Nav 1.5 & $\begin{array}{c}\text { CACCTTCACCGC } \\
\text { CATCTACA }\end{array}$ & $\begin{array}{l}\text { AAGGTGCGTAAG } \\
\text { GCTGAGAC }\end{array}$ \\
\hline CACNA1C & Cav 1.2 & $\begin{array}{c}\text { CCTCGAAGCTGG } \\
\text { GAGAACAG }\end{array}$ & $\begin{array}{c}\text { TGTGTGGGAGTC } \\
\text { AATGGAGC }\end{array}$ \\
\hline KCNJ2 & Kir 2.1 & $\begin{array}{c}\text { GACGCCTTCATC } \\
\text { ATTGGTGC }\end{array}$ & $\begin{array}{c}\text { CCGGACATGAGC } \\
\text { TTCCACAA }\end{array}$ \\
\hline GJA5 & Con 40 & $\begin{array}{c}\text { ATACCATTCAGC } \\
\text { CTGGTTGC }\end{array}$ & $\begin{array}{c}\text { GGTGGGCCTCTT } \\
\text { TAGCTTTC }\end{array}$ \\
\hline GJA1 & Con 43 & $\begin{array}{l}\text { GGACTGCTTCCT } \\
\text { CTCACGTC }\end{array}$ & $\begin{array}{c}\text { CAGCTTGTACCC } \\
\text { AGGAGGAG }\end{array}$ \\
\hline GJA7 & Con 45 & $\begin{array}{c}\text { TTTGTGTGCAAC } \\
\text { ACAGAGCA }\end{array}$ & $\begin{array}{c}\text { GGTCCTCTTCCG } \\
\text { TTTCTTCC }\end{array}$ \\
\hline 36B4 & & $\begin{array}{c}\text { GCGACCTGGAAG } \\
\text { TCCAACTA }\end{array}$ & $\begin{array}{c}\text { ATCTGCTGCATC } \\
\text { TGCTTGG }\end{array}$ \\
\hline \multicolumn{2}{|c|}{ CYCLOPHYLIN } & $\begin{array}{c}\text { ACAGGTCCTGGC } \\
\text { ATCTTGTC }\end{array}$ & $\begin{array}{c}\text { CATGGCTTCCAC } \\
\text { AATGTTCA }\end{array}$ \\
\hline
\end{tabular}

\subsection{Gene expression analysis}

In order to evaluate the effects of the cell culture substrate on main ion channels gene expression, RNA from three samples in each substrate was isolated using Tri-reagent (Sigma). Transcripts were quantified in a twostep RT-PCR. First strand cDNA was synthesized using High Capacity cDNA Reverse Transcription Kit (Applied Biosystems). The samples were later run using SYBR Green oligonucleotides and the CFX Real Time PCR detection Systems (Bio Rad). Analysis was performed with two technical replicates for each sample. Gene expression values were normalized to two standard housekeeping genes (36b4 and Cyclophylin) as internal controls, and expressed as relative mRNA levels (relative expression). Primer sequences are shown in Table 1.

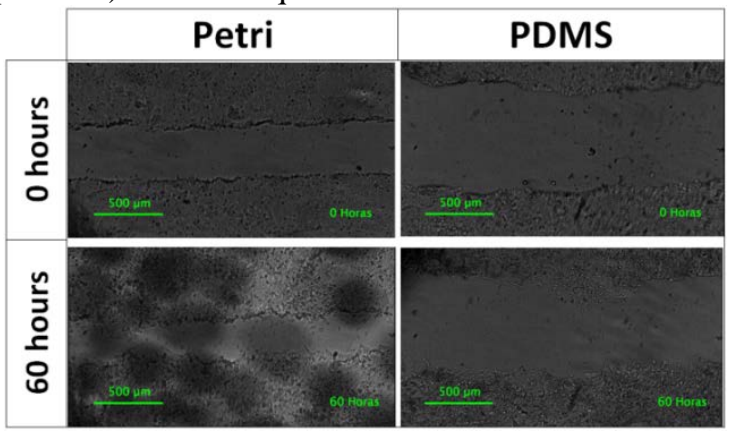

Figure 2. Wound test images in both substrates. 


\section{Results}

\subsection{Proliferation}

HL-1 cells were able to grow, attach and keep spontaneous contraction over both substrates. In fact, proliferation was high because of the cell tumorous origin [4], as $100 \%$ of reduction was achieved within 6 days. Although not significant differences were observed, in Petri highest proliferation rates occurred at early stages of the cell culture, whereas in PDMS at late stages. Moreover, in Petri dishes a more reproducible response was observed, as in PDMS wells standard deviation between the three samples was considerably greater.

\subsection{Migration and displacement}

The ability of HL-1 cells to close induced wounds was demonstrated to be limited over both substrates, as it is characteristic of adult cardiomyocytes. After 60 hours, none of the injuries were completely closed in any of the materials (Figure 2). The analysis of maximum displacement velocities (i.e. $0.40 \mu \mathrm{m} / \mathrm{min}$ in PDMS and $0.25 \mu \mathrm{m} / \mathrm{min}$ in Petri) demonstrated the reduced ability to migrate of these adult cardiomyocytes suggesting that these cells colonize only by proliferation.

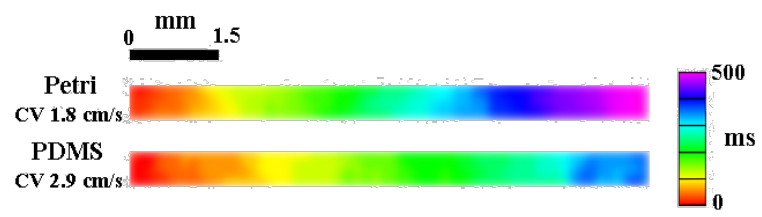

Figure 3. Isochrones maps of a significant band in each substrate.

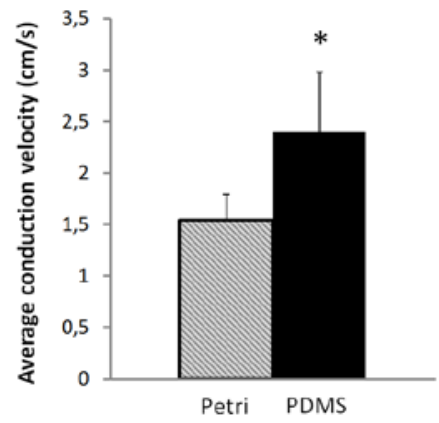

Figure 4. Average conduction velocity for each substrate. $\mathrm{p}<0.05(*)$

\subsection{Conduction velocity}

A representative example of isochrones maps for Petri and PDMS generated from the optical mapping movies data is shown in Figure 3. Uniform impulse propagation can be observed in both bands, confirming that the cells were confluent and that conduction followed a straight path. Notice that the time impulse takes to reach the end of the band in the PDMS well is shorter than in the Petri dish, suggesting faster conduction velocities.

Average conduction velocity values of HL-1 cells grown in Petri and PDMS can be observed in Figure 4. Impulse propagated significantly faster when cells were grown in flexible membranes as the conduction velocity was $156 \%$ greater in PDMS wells $(2.4 \mathrm{~cm} / \mathrm{s})$ than in Petri dishes $(1.5 \mathrm{~cm} / \mathrm{s})$.

\subsection{Gene expression analysis}

The gene expression analysis supported what was observed at a functional level, as genes analysed codifying for proteins involved in action potential generation and propagation were higher expressed in cells grown over PDMS wells (

Figure 5).

A higher expression of genes codifying for molecular components of sodium, calcium and potassium channels (SCN5A, CACNA1C and KCNJ) and of genes codifying for connexins forming part of gap junctions (GJA5, GJA1, GJA7), indicate a greater presence of these proteins in HL-1 cells.

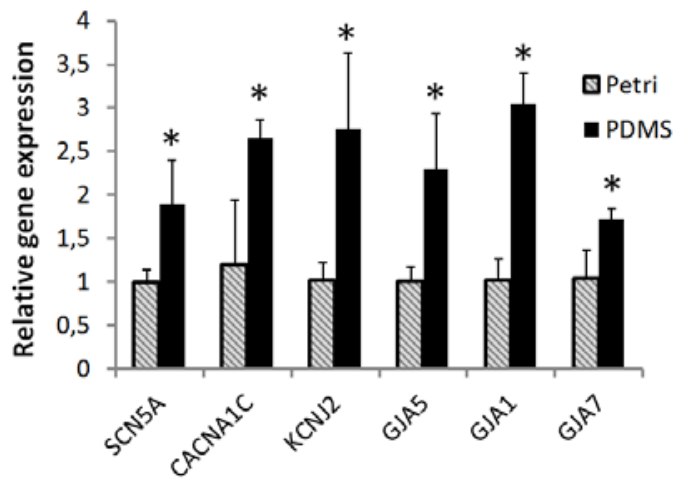

Figure 5. Expression levels of ion channels in HL-1 cells grown in PDMS versus Petri substrates. SCN5A, CACNA1C and KCNJ2 genes codify for proteins which are subunits of ion channels associated to $\mathrm{I}_{\mathrm{Na}}, \mathrm{I}_{\mathrm{CaL}}$ and $\mathrm{I}_{\mathrm{K} 1}$ respectively. GJA5, GJA1, GJA7 genes codify for components of the gap junctions: connexin 40, connexin 43 and connexin 45 respectively. Differences are statistically significant when $\mathrm{p}<0.05$ (*) (TTest).

\section{Discussion}

\subsection{Main contributions}

The results of this study demonstrate that the culture of cardiac cells on flexible membranes has an impact on their phenotype. This impact has shown to be especially significant in electrophysiological properties, as 
conduction velocity has increased considerably. The similar flexibility of PDMS with that of the ECM may be the reason behind the gene expression profile more characteristic of adult cardiomyocytes. These changes in gene expression may have led to a higher presence of gap junctions and ion channels that ease impulse generation and propagation.

The use of flexible membranes opens new insights into cardiac patches for cardiac regeneration, as obtaining higher conduction velocities while maintaining other general cell properties is possible. As impulses propagate faster, the possibility of future re-entries will diminish and the patch will integrate better within healthy tissue. Flexible membranes also allow future studies of electrophysiological properties during cell culture under simultaneous mechanical and electrical stimulation, which is otherwise impossible with rigid Petri dishes. These conditions may improve characteristic cardiomyocyte properties even further, leading to better quality patches.

\subsection{Applications}

The generation of cardiac patches that mimic in vivo properties have two main applications: (1) as therapy for cardiac regeneration of scar tissue after an infarct and (2) as models of cardiac tissue for in-vitro drug and therapy testing.

In order to serve as therapy, one of the main limitations is low conduction velocity values in cardiac patches. In addition to that, cells which present high proliferation and migration capacity are required in order to populate the patches effectively. To obtain considerable migration capacity, immature cardiomyocytes such as cardiopoietic cells or iPSCs not terminally differentiated into cardiomyocytes should be contemplated. To reach higher conduction velocity values these immature cells should be further differentiated.

HL-1 cells grown over flexible membranes present better simulation of cardiac tissue dynamics as conduction velocities are closer to in vivo values. Low migration capacity observed, also mimicks accurately adult cardiomyocyte behaviour.

\subsection{Limitations}

The results obtained for conduction velocity measurements, although significantly higher for flexible membranes, are still far from in vivo impulse propagation velocities, which may be due to the limited number of cells interconnected in a monolayers. The use three dimensional in vitro structures such as decellularized matrices could contribute to the improvement of cardiomyocytic properties, as it provides an ideal environment for cells to grow [6].

\section{Conclusions}

The use of flexible membranes has shown to play a role in cardiac cell phenotype, especially inducing better impulse propagation. At the molecular level, expression of genes codifying for proteins involved in ion channels and gap junctions were also more expressed in flexible membranes, suggesting that this may be the cause of the higher conduction velocity measurements.

\section{Acknowledgements}

Supported in part by grants from the Instituto de Salud Carlos III and Ministry of Economy and Competitiveness of Spain: (Grants PI13-01882, PI13-00903 and PI14/00857, Red RIC, PLE2009-0152 and Red de Terapia Celular); Spanish Society of Cardiology (Grant for Clinical Research in Cardiology 2015); Spanish Ministry of Economy and Competitiveness, Spain (Grant: IJCI-2014-22178 and TEC2013-50391-EXP).and by Becas Santander de Prácticas en PYMES.

\section{References}

[1] Gaziano T, Reddy KS, Paccaud F, et al. Cardiovascular Disease. Disease Control Priorities in Developing Countries. 2nd ed. Washington (DC): World Bank; 2006.

[2] Rubio Guivernau JL, Ledesma Carbayo MJ, Arenal Maíz Á, et al. Do the spatial characteristics of myocardial scar tissue determine the risk of ventricular arrhythmias? Cardiovasc Res. 2012 Mar 1;94(10.1093/cvr/cvs113):32432.

[3] Wang B, Borazjani A, Tahai M, et al. Fabrication of cardiac patch with decellularized porcine myocardial scaffold and bone marrow mononuclear cells. J Biomed Mater Res A. 2010 Sep 15;94(4):1100-10.

[4] Claycomb WC, Lanson NA, Stallworth BS, et al. HL-1 cells: a cardiac muscle cell line that contracts and retains phenotypic characteristics of the adult cardiomyocyte. Proc Natl Acad Sci U S A. 1998 Mar 17;95(6):2979-84.

[5] Climent AM, Guillem MS, Fuentes L, et al. Role of atrial tissue remodeling on rotor dynamics: an in vitro study. Am J Physiol Heart Circ Physiol. 2015 Dec 1;309(11):H196473

[6] Sánchez PL, Fernández-Santos ME, Costanza S, et al. Acellular human heart matrix: A critical step toward whole heart grafts. Biomaterials. 2015 Aug;61:279-89.

Address for correspondence.

Andreu M. Climent

Laboratorio de órganos y matrices bioartificiales

Instituto de Investigación Sanitaria Gregorio Marañón

Hospital GU Gregorio Marañón. Edificio Materno Infantil.

c) O'Donnell 48,

28009, Madrid, Spain

andreu.climent@gmail.com 